\title{
Delivery of neostigmine and glycopyrrolate by iontophoresis: a nonrandomized study in individuals with spinal cord injury
}

\author{
Mark A. Korsten ${ }^{1,2,3} \cdot$ Brian L. Lyons $^{1} \cdot$ Miroslav Radulovic $^{1,2} \cdot$ Tradd M. Cummings $^{1} \cdot$ Gautam Sikka $^{2,3}$. \\ Kamaldeep Singh $^{1,2} \cdot$ Joshua C. Hobson ${ }^{1} \cdot$ Anton Sabiev $^{1}$ - Ann M. Spungen $\mathbb{B}^{1,3,4} \cdot$ William A. Bauman ${ }^{1,2,3,4}$
}

Received: 17 May 2017 / Revised: 18 September 2017 / Accepted: 22 September 2017 / Published online: 8 November 2017

(c) International Spinal Cord Society 2018

\begin{abstract}
Study design Phase I Clinical Trial.

Objectives In this proof-of-principle study, the effectiveness and safety of transdermal administration of neostigmine/ glycopyrrolate to elicit a bowel movement was compared to intravenous administration in patients with spinal cord injury. Setting James J. Peters Veterans Affairs Medical Center (Bronx, NY).

Methods Individuals were screened for responsiveness (Physical Response) to intravenous neostigmine $(0.03 \mathrm{mg} / \mathrm{kg}) / \mathrm{gly}-$ copyrrolate $(0.006 \mathrm{mg} / \mathrm{kg})$. Intravenous neostigmine/glycopyrrolate responders (Therapeutic Response) were administered low-dose transdermal neostigmine $/$ glycopyrrolate $[(0.05 \mathrm{mg} / \mathrm{kg}) /(0.01 \mathrm{mg} / \mathrm{kg})]$ by iontophoresis. Non-responders to lowdose transdermal neostigmine/glycopyrrolate were administered high-dose transdermal neostigmine/glycopyrrolate $[(0.07$ $\mathrm{mg} / \mathrm{kg}) /(0.014 \mathrm{mg} / \mathrm{kg})]$ by iontophoresis. Bowel movement, bowel evacuation time, and cholinergic side effects were recorded. Visits were separated by 2 to 14 days.

Results Eighteen of 25 individuals $(72.0 \%)$ had a bowel movement $(20 \pm 22 \mathrm{~min})$ after intravenous neostigmine/glycopyrrolate. Of these 18 individuals, 5 individuals experienced a bowel movement with low-dose transdermal neostigmine/ glycopyrrolate. Another five individuals had a bowel movement after high-dose transdermal neostigmine/glycopyrrolate administration. Fewer side effects were observed in individuals who received neostigmine/glycopyrrolate transdermally compared to those who were administered intravenous neostigmine/glycopyrrolate.

Conclusions Transdermal administration of neostigmine/glycopyrrolate by iontophoresis appears to be a practical, safe, and effective approach to induce bowel evacuation in individuals with spinal cord injury.
\end{abstract}

\section{Introduction}

Spinal cord injury (SCI) is associated with a slowing of intestinal peristaltic activity, which is most likely the result

Mark A. Korsten

mark.korsten@va.gov

1 National Center for the Medical Consequences of Spinal Cord Injury, Bronx, NY, USA

2 Medical Service, James J. Peters VA Medical Center, Bronx, NY, USA

3 Department of Medicine, The Icahn School of Medicine at Mount Sinai, New York, NY, USA

4 Department of Rehabilitation Medicine, The Icahn School of Medicine at Mount Sinai, New York, NY, USA of down-regulation of parasympathetic neural pathways leading from the central nervous system to the gut [1]. Neurogenic bowel is common after SCI and is characterized by difficulty with evacuation, abdominal pain, and fecal incontinence. Management of this condition is often timeconsuming and unpleasant, and despite current practices, such as dietary manipulation, laxative administration, and ano-rectal instillation of cathartics, bowel dysfunction remains a major source of anxiety and morbidity. As a result, more efficacious approaches to treat neurogenic bowel are needed.

Parasympathetic stimulation of the bowel accelerates peristalsis and improves bowel care [2]. By its cholinergic action, neostigmine (NEO) initiates high amplitude peristaltic contractions in the recto-sigmoid colon that are typically absent in persons with SCI [3, 4]. However, because of the potential cardiopulmonary toxicity of NEO, 
it has been administered in combination with a selective anticholinergic agent, glycopyrrolate (GLY). In our prior studies, GLY administration has been found to selectively block the cardiopulmonary side effects of NEO without significantly attenuating the pro-kinetic peristaltic response of the colon ${ }^{3}$. The efficacy of this dual drug combination in improving bowel preparations for elective colonoscopy has also been described by our group [5].

To date, intravenous (IV), intramuscular, and subcutaneous administration of NEO/GLY has been reported by our group [3-5]. Parenteral administration routes for these drugs are impractical for long-term bowel care in the SCI population. Other methods of delivery have been attempted, including sublingual and intranasal administration, but neither of these alternative approaches proved useful, possibly because the mucosal surfaces are lined by epithelial cells whose cell membranes are permeable only to small uncharged solutes.

Iontophoresis (ION) is a noninvasive technique that uses a low-intensity electric current to drive charged substances across the skin. This approach has been used with a number of drugs, specifically charged proteins, peptides, and oligonucleotides [6-8]. Since NEO/GLY are both positively charged compounds and are smaller than $500 \mathrm{kDa}$, the transdermal (TD) delivery of these molecules by ION appeared to be a feasible therapeutic delivery route for longterm bowel care. In order to deliver a positively charged drug, it requires placement under the positively charged electrode (anode), which repels the drug that is simultaneously attracted to the negative electrode (cathode) which is placed in a proximate anatomical location on the skin. Chemical methods are also potentially useful to enhance the penetration of the medication through the stratum corneum to achieve heightened drug absorption and improved systemic effect. A large body of literature exists supporting the use of fatty acids in order to increase permeability of the skin [9-12].

Optimal bowel care is often lacking in patients with SCI and other neurogenic diseases despite multiple drugs and lifestyle modifications. It is hypothesized that TD administration of NEO + GLY is a safe and efficacious alternative for long-term bowel care in SCI patients with bowel movement (BM) difficulty.

\section{Methods}

Veterans and non-Veterans from the greater New York City area with SCI, who have been identified to have neurogenic bowel, were recruited for the study and were not excluded from participation based on their status (inpatient/outpatient) in the VA hospital. This prospective, nonrandomized study was approved by the Institutional
Review Board of the James J. Peters VA Medical Center (JJPVAMC) and the Food and Drug Administration (FDA) (IND 117034). The study was registered on the Clinical Trials.gov website (ClinicalTrials.gov Identifier: NCT02370862).

During the two-year study, 25 individuals were screened, 4 were excluded, and none denied consent. Individuals of both genders, between 18 and 75 years of age, with chronic $\mathrm{SCI}$ (SCI for $\geq 1$ year) and who required $1 \mathrm{~h}$ or greater to perform bowel care were eligible for study enrollment. Due to contraindications specific to the administration of NEO and GLY, individuals were excluded from the study if they had any history of cardiovascular disease (i.e. myocardial infarction, bradycardia, and cardiac arrhythmias), mechanical obstructions of the intestinal tract, and/or pulmonary disease (i.e. asthma or chronic obstructive pulmonary disease). All individuals signed an informed consent agreeing to voluntarily participation in this study. All of the procedures and data collection were performed at the James J. Peters VA Medical Center, Bronx, New York.

Determining the efficacy of transdermal NEO/GLY delivered via iontophoresis to promote a $\mathrm{BM}$ in individuals with chronic SCI is the primary objective being evaluated. Safety and tolerability of TD NEO/GLY in these individuals, when used to promote a BM, was the secondary objective, and was quantified through the monitoring of the side effects. In order to assess the degree of responsiveness to this drug combination, all individuals were first screened with IV NEO/GLY. The doses for IV administration were dependent on body mass as follows: NEO $(0.03 \mathrm{mg} / \mathrm{kg})$ and GLY $(0.006 \mathrm{mg} / \mathrm{kg})$. For $60 \mathrm{~min}$ following administration of IV NEO/GLY, blood pressure, heart rate, and pulse oximetry $\left(\mathrm{SPO}_{2}\right)$ were monitored continuously. Patients were assessed for the cholinergic side effects of NEO, including those of ocular/facial muscular twitching, diaphoresis, increased or decreased salivation, and abdominal distension. Airway resistance was measured using an impulse oscillometry system (IOS) pre- and post-administration at both, the low frequency (R5) and high frequency (R20) to estimate total and central pulmonary resistances, respectively, and to calculate peripheral airway resistance (R5-R20). A physician was present monitoring cardiopulmonary changes following drug administration. Individuals who had positive response to IV NEO/GLY (i.e., BM and/or self-reported cholinergic symptoms) were eligible to receive two consecutive visits during which a dose titration for the administration of NEO/GLY was performed by TD-ION.

Individuals were asked to abstain from bowel care for at least $24 \mathrm{~h}$ prior to the study, increasing the likelihood that stool would be present in the left colon and rectum. During the first visit, individuals received placebo (e.g., normal saline) and then after $60 \mathrm{~min}$ 'low' dose NEO $(0.05 \mathrm{mg} / \mathrm{kg})$ 
and GLY $(0.01 \mathrm{mg} / \mathrm{kg})$. Individuals who did not report a BM and/or cholinergic symptoms following the administration of the low-dose NEO/GLY had the second visit with the administration of 'high' dose NEO $(0.07 \mathrm{mg} / \mathrm{kg})$ and GLY $(0.014 \mathrm{mg} / \mathrm{kg})$. For the purposes of this study, the maximal dose approved by the FDA was NEO $10.0 \mathrm{mg}$ and GLY $2.0 \mathrm{mg}$ per subject. The end points for transdermal administration were identical to those recorded after the IV administration, specifically BM within $60 \mathrm{~min}$ of ION and bowel evacuation time (BET)

The ION technology consisted of 2 Dynatron iBoxes (Salt Lake City, UT). These iBoxes delivered an electric current of $4.0 \mathrm{~mA} / \mathrm{min} \times 10 \mathrm{~min}$ via anode electrode patches, with a maximum medicated solution volume of 4.0 $\mathrm{ml}$. Due to the weight of individuals, as well as the volume limitation of our electrodes, each study medication was placed on two separate anode electrodes and applied onto the quadriceps and calf muscles of the patients. The cathodes (return electrodes) were applied on the inner knees of individual approximately $6 \mathrm{in}$. away from and mid-way between the anode electrodes. The skin under the anodes was pretreated with oleic acid to enhance the drug permeation through the skin barrieR [11].

The primary outcome of a BM was measured using a timer initiated at the beginning of the drug administration, be it via IV or TD-ION routes, and ending at first indications (auditory or visual) of stool in the commode or toilet. Side effects, also noted to determine responsiveness to the drug, were reported by the participant or the researcher. Cholinergic hyperactivity response to the NEO was

Table 1 Characteristics of SCI study population

\begin{tabular}{ll}
\hline Age (years) & $50 \pm 15$ \\
Height $(\mathrm{cm})$ & $180 \pm 9.1$ \\
weight $(\mathrm{kg})$ & $87.3 \pm 16.9$ \\
BMI $\left(\mathrm{kg} / \mathrm{cm}^{2}\right)$ & $26.8 \pm 4.3$ \\
DOI (years) & $9.0 \pm 9.0$ \\
Para $n(\%)$ & $15(60 \%)$ \\
Tetra $n(\%)$ & $10(40 \%)$ \\
Neurological Injury Level & Cervical $(n=11)$, thoracic \\
Distribution & $(n=13)$, lumbar $(n=1)$ \\
ASIA Impairment Scale & A $(n=12), \mathrm{B}(n=3), \mathrm{C}(n=7)$, \\
Distribution & $\mathrm{D}(n=3)$
\end{tabular}

Data are prsented as mean \pm standard deviation. ASIA American Spinal Injury Association, $A$ complete (no sensory or motor function below level of injury), $B$ sensory incomplete (sensory but no motor function below neurological level of injury), $C$ motor incomplete (motor function preserved below neurological level and sacral region; $50 \%$ of muscles below level of injury cannot move against gravity), $D$ motor incomplete (greater motor function preserved below neurological level and sacral region; more than $50 \%$ of muscles can move against gravity), BMI body mass index, $\mathrm{cm}$ centimeters, DOI duration of injury, $k g$ kilograms, $n$ number of subjects, para paraplegia, tetra teraplegia recorded if signs or symptoms such as: eye lid/facial twitching, abdominal distention, cramping, dry mouth, diaphoresis, drowsiness, lightheadedness, or headache has occurred. Safety being the secondary outcome variable monitored using a cardiac recorder evaluating changes in blood pressure, heart rate, and $\mathrm{SPO}_{2}$.

Demographic continuous variables are reported as mean \pm SD. Nonparametric independent samples Kruskal-Wallis analysis was used to determine significant differences among the compared conditions (IV, Low dose, High dose) for blood pressure, heart rate, $\mathrm{SPO}_{2}$, and impulse oscillometry values. A chi-square analysis was used to determine the significance of the percentage of BMs in IV and TD groups. Among the three different dosing conditions, differences were determined for BET, reported side effects from the patient, and clinically relevant changes in blood pressure [ $>30 \mathrm{mmHg}$ drop in mean arterial pressure], heart rate $\left(>25 \%\right.$ decrease), and $\mathrm{SPO}_{2}(>5 \%$ decrease) [using cardiac recorder] following drug administration. The level of significance was set at $p<0.05$ for all analyses. Multiple BMs have not been recorded.

\section{Results}

Twenty-five individuals with SCI were recruited to receive IV NEO/GLY. The baseline demographic characteristics of the recruited SCI population and BET are provided (Tables 1 and 2). Eighteen of these 25 (72\%) individuals had experienced a BM. Of the remaining four non-responders, three individuals experienced symptoms indicative of a NEO/GLY effect without a BM. All 21 individuals, who responded to the IV NEO/GLY, received TD low-dose $\mathrm{NEO} / \mathrm{GLY}$ and 5 responded with a BM within $60 \mathrm{~min}$. Of the 16 individuals who did not respond to low-dose NEO/ GLY, 5 individuals had a BM when given high-dose TD NEO/GLY (Fig. 1). However, there were no significant

Table 2 Reported side effects

\begin{tabular}{llll}
\hline Side effects & IV & Low TD & High TD \\
\hline Eye/Facial Twitching* & $23 / 25(92 \%)$ & $2 / 21(10 \%)$ & $4 / 16(25 \%)$ \\
Abdominal distention & $10 / 25(40 \%)$ & $4 / 21(19 \%)$ & $7 / 16(44 \%)$ \\
Abdominal cramping & $13 / 25(52 \%)$ & $4 / 21(19 \%)$ & $5 / 16(31 \%)$ \\
Dry mouth & $14 / 25(56 \%)$ & $5 / 21(24 \%)$ & $2 / 16(13 \%)$ \\
Diaphoresis & $8 / 25(32 \%)$ & $3 / 21(14 \%)$ & $3 / 16(19 \%)$ \\
Drowsiness & $6 / 25(24 \%)$ & $4 / 21(19 \%)$ & $1 / 16(6 \%)$ \\
Lightheadedness $^{\ddagger}$ & $7 / 25(28 \%)$ & $2 / 21(10 \%)$ & 0 \\
Headache** $^{4 / 25(16 \%)}$ & 0 & 0 \\
\hline
\end{tabular}

Data reported as number and percentage of subjects reporting the defined side effects. All comparisions are an analysis of variance between all three groups. $I V$ Intravenous, $T D$ transdermal dose.

$* p<0.001 ;{ }^{\dagger} p=0.006 ;{ }^{¥} p=0.029 ; * * p=0.039$ 


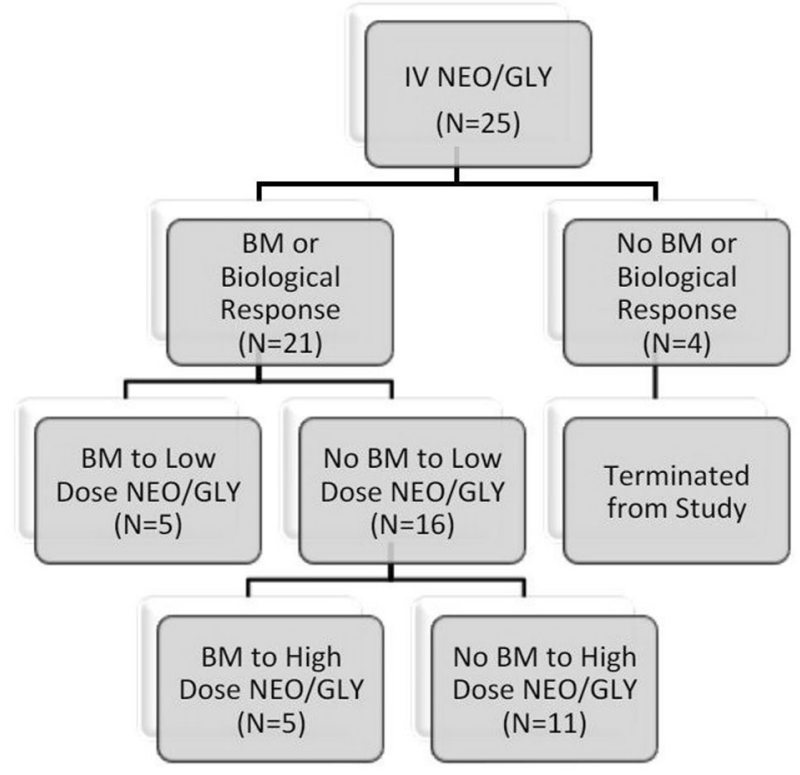

Fig. 1 Study flow diagram. Depiction of subject response and study progression. $B M$ bowel movement, $G L Y$ glycopyrrolate, $I V$ intravenous, $N O E$ neostigmine

differences in BET among the three doses of drug [21 \pm 23 $\min$ vs. $36 \pm 15 \mathrm{~min}$ vs. $26 \pm 22 \mathrm{~min}(\mathrm{~F}=1.05 p=0.37)]$. Regarding intention to treat, a BM was observed in 10 of the original 25 individuals $(40 \%)$ when combining the findings of the response to high-dose and low-dose TD NEO/GLY administration. This response rate to TD administration was lower $(p=0.083)$ than the response rate to IV NEO/GLY (72\%). Of the 21 responders to the IV administration of NEO/GLY, a BM was observed in $48 \%$ (10 of 21) in the combined response to high- and low-dose TD-ION administration of NEO/GLY. No individuals experienced responsiveness to TD placebo administration. No difference in BET or frequency of BM between the individuals neurogenic bowel due to the upper or lower motor neuron injury has been observed.

All groups reported the anticipated cholinergic side effects following drug administration (Table 2). However, individuals who received IV NEO/GLY experienced a greater number of side effects compared to TD administrations: eye/facial twitching $(p<0.001)$; lightheadedness $(p=0.029) ;$ headache $(p=0.039)$, and dry mouth $(p=0.006)$. Symptoms were considered tolerable by all individuals. No serious cardiopulmonary side effects were detected and none required medical intervention or administration of an anticholinergic agent.

Individuals receiving IV NEO/GLY demonstrated significantly greater change in mean arterial pressure when compared to combined TD groups at the 5 min interval $(p=0.008))$. In the low-dose TD group, one subject had mean arterial pressure changes: no patient receiving high- dose TD NEO/GLY experienced any hemodynamic changes at the 5 min time point. No significant changes in mean arterial pressure were observed in either group at any other time points during the $60 \mathrm{~min}$ of monitoring after drug administration. Significant systolic blood pressure changes with IV NEO/GLY administration were also measured at the $5 \mathrm{~min}(p=0.021)$ and the $15 \mathrm{~min}$ intervals $(p=0.047)$. There were no other clinically significant changes in heart rate or $\mathrm{SPO}_{2}$ among the three doses of drug. In addition, bronchoconstriction was not observed but, rather, slight bronchodilation or no significant change following administration of the drug combination, as measured by percent change in $\mathrm{R} 5(\mathrm{kPa} / \mathrm{l} / \mathrm{s})$ frequency $[-3.75 \pm 33.4 \mathrm{vs}$. $-2.55 \pm 16.9$ vs. $3.53 \pm 17.1 \quad(\mathrm{~F}=0.42 p=0.66)]$ and $\mathrm{R} 20(\mathrm{kPa} / \mathrm{l} / \mathrm{s})$ frequency $[-6.76 \pm 30.6$ vs. $0.100 \pm 18.3 \mathrm{vs}$. $1.73 \pm 16.9(\mathrm{~F}=0.704 p=0.50)]$.

\section{Discussion}

TD NEO/GLY resulted in BMs in individuals with SCI complicated by neurogenic bowel. This proof-of-principle study demonstrated that TD NEO/GLY, administered at FDA-approved study doses, successfully stimulates BMs in a subgroup of patients with chronic SCI. In addition, there were fewer side effects observed after TD NEO/GLY than after IV NEO/GLY.

ION has therapeutic value in a number of clinical settings. ION has achieved clinical relevance in treatment of musculoskeletal conditions, such as fasciitis, bursitis, and epicondylitis [13]. ION approach has also been approved by the FDA for the treatment of migraines using sumatriptan [14], as well as to manage the postoperative pain with fentanyl [15]. Our study is the first of its kind to demonstrate a potential role for ION in the administration of a drug that stimulates bowel contraction and stool evacuation.

The primary endpoint of our study, a BM following TD and IV administration of NEO/GLY, differed by $24 \%$ between the two routes ( $48 \%$ vs. $72 \%$ ). However, due to the difficulties and dangers inherent in frequent or semipermanent venous access, such as thrombosis, infection, and bleeding ${ }^{16}$, TD administration of NEO/GLY has practical clinical advantages ${ }^{17}$ in non-hospitalized individuals with long-term bowel care requirements. Sample size of this and of our other studies are small because of the wellknown difficulty in recruiting SCI individuals for bowelrelated investigation, further diminished by the application of stringent exclusion criteria for safety reasons. As a result, the power of our study is too low to designate the $24 \%$ difference in BM between TD and IV routes as statistically significant. However, the greater rate of cholinergic effects observed after IV administration of NEO/GLY suggests that the IV route is more effective than the TD route at triggering 
a BM. In any case, ION NEO/GLY does not need to be superior or even comparable to IV NEO/GLY, in order to be a useful from a clinical standpoint as an alternate delivery method, especially in domestic use ${ }^{18}$. A higher dose of NEO with ION administration may be speculated to have resulted in higher blood levels of the drug, thus improving the likelihood of evacuation.

It is possible, that the degree of skin permeability of those who have failed to have BMs was lower, altering the kinetics of NEO absorption. Differential absorption of NEO could also be attributed to variability in stratum corneum thickness between individuals. In addition, the optimal ratio of NEO to GLY may vary among individuals. Some individuals may have greater sensitivity to the anticholinergic actions of GLY, thereby lessening the cholinergic effects of NEO on the colon. Non-responders that reported sensations indicative of increased bowel peristalsis without a BM could possibly be due to the lack of stool in the colon or non-propulsive peristaltic waves. The pro-kinetic actions of NEO are likely mediated by the M2 type of muscarinic acetylcholine receptors in the colon ${ }^{19}$, the activity of which may have been blocked by GLY within some individuals who happen to have M2 receptors with a higher affinity to GLY diminishing the likelihood of a BM, which could also account for some of the non-responders to IV NEO/GLY.

In some individuals, NEO may have caused inadvertent contraction of the anal sphincter, or there may have been an existing state of spasm of the anal sphincter as a result of neurological injury. This was noted in a number of individuals regardless of administration method. In such patients, digital rectal stimulation might improve the likelihood of a BM following NEO administration. It is possible that a larger TD dose and/or augmentation of factors that increase uptake, and, in select cases altering the ratio of NEO to GLY, would increase the therapeutic efficacy of TD NEO/ GLY, along with consideration of performing a mechanical maneuver to relax the anal sphincter to permit the expulsion of stool.

Though no serious side effects requiring reversal agents were observed in our study, bradycardia and bronchoconstriction are known serious side effects of anticholinergic agents. Differences in bronchial resistance at the total (R5) and central (R20) oscillation frequencies, from before to after the medication administration, were statistically insignificant. It is important to re-emphasize that patients with a history of coronary artery disease, obstructive airway disease, or bradycardia were excluded from the study. One should not consider NEO/GLY to be risk-free even in healthy individuals until the safety of the drug combination has been demonstrated in a given individual. We recommend that the initial administration of this drug combination be performed in a monitored setting.
In summary, TD administration of NEO/GLY via ION appears to be a practical, safe, and effective approach to induce BMs in individuals with SCI.

Acknowledgments We thank Ms. Oriana Tascione, research coordinator in the NCMSCI, who assisted in subject recruitment and protocol implementation; Dr. Ravi Vinnakota, who assisted in the oversight of neostigmine and glycopyrrolate administration; Dr. Marinella Galea, a staff physician in Spinal Cord Services; and Dr. Sandeep Kahal who have assisted in subject recruitment.

Author Contributions M.A.K. Chief of Gastroenterology and Invasive Gastroenterology, is the guarantor of this submission. M.A.K. was primarily responsible for protocol design and implementation. B.L.L. research coordinator in the National Center for the Medical Consequences of Spinal Cord Injury (NCMCSCI), was responsible for subject recruitment, protocol implementation, and assisted with the preparation of this manuscript. M.R. a Veterans Affairs Career Development Awardee, oversaw administration of neostigmine and glycopyrrolate. T.C. research coordinator in the NCMCSCI, assisted with the preparation of this manuscript, statistical analysis of the data, and subject recruitment. G.S. performed statistical analysis of the data. K.S. assisted in subject recruitment and protocol implementation. J.H. research coordinator in the NCMCSCI, assisted in subject recruitment and protocol implementation. A.M.S. the Associate Director of NCMCSCI, assisted with the protocol design, and along with A.S. worked on the statistical analysis and the writing of this manuscript. W.A.B. the Director of NCMCSC, was responsible for the conceptualization of this project with M.A.K. and assisted in the preparation of the protocol and the manuscript. All authors listed on this study have read and approved the final version being submitted for consideration of publication.

Funding This research was supported by the Department of Veterans Affairs Rehabilitation Research and Development Service (\#B9212-C) and the James J. Peters VA Medical Center.

\section{Compliance with ethical standards}

Conflict of interest M.A.K. and W.A.B. are co-inventors of this bowel intervention and have processed a patent (patent number: 7.635,709) and a provisional patent $(62 / 2984,874)$ for the administration of neostigmine and glycopyrrolate via iontophoresis through the Office of Technology Transfer Program, Department of Veterans Affairs. M.A. K. reports grants from Department of Veteran Affairs Rehabilitation Research and Development Service, during the conduct of the study; In addition, M.A.K. has a patent 7.635,709 licensed to Technology Transfer Program of the Department of Veterans Affairs. W.A.B. reports grants from Department of Veteran Affairs Rehabilitation Research and Development Service, during the conduct of the study; In addition, W.A.B. has a patent 7.635,709 licensed to Technology Transfer Program of the Department of Veterans Affairs.

\section{References}

1. Fajardo NR, Pasiliao RV, Modeste-Duncan R, Creasey G, Bauman WA, Korsten MA . Decreased colonic motility in persons with chronic spinal cord injury. Am J Gastroenterol. 2003;98:128-34.

2. Altaf MA, Sood MR. The nervous system and gastrointestinal function. Dev Disabil Res Rev. 2008;14:87-95. 
3. Korsten MA, Rosman AS, Ng A, Cavusoglu E, Spungen AM, Radulovic $M$ et al. Infusion of neostigmine-glycopyrrolate for bowel evacuation in persons with spinal cord injury. Am J Gastroenterol. 2005;100:1560-5.

4. Rosman AS, Chaparala G, Monga A, Spungen AM, Bauman WA, Korsten MA Intramuscular neostigmine and glycopyrrolate safely accelerated bowel evacuation in patients with spinal cord injury and defecatory disorders. Dig Dis Sci. 2008;53:2710-3.

5. Korsten MA, Spungen AM, Radulovic M, Rosman AS, Hunt K, Galea MD Neostigmine administered with moviprep improves bowel preparation for elective colonoscopy in patients with spinal cord injury: a randomized study. J Clin Gastroenterol. 2015;49:751-6.

6. Dhote V, Bhatnagar P, Mishra PK, Mahajan SC, Mishra DK. Iontophoresis: a potential emergence of a transdermal drug delivery system. Sci Pharm. 2012;80:1-28.

7. Dixit N, Bali V, Baboota S, Ahuja A, Qureshi J. Iontophoresisan approach for controlled drug delivery: a review. Curr Drug Deliv. 2007;4:1-10.

8. Sieg A, Jeanneret F, Fathi M, Hochstrasser D, Rudaz S, Veuthey $\mathrm{JL}$ et al. Extraction of amino acids by reverse iontophoresis in vivo. Eur J Pharm Biopharm. 2009;72:226-31.

9. Nair VB, Panchagnula R. Effect of iontophoresis and fatty acids on permeation of arginine vasopressin through rat skin. Pharmacol Res. 2003;47:563-9.

10. Pillai O, Panchagnula R. Transdermal iontophoresis of insulin. VI. Influence of pretreatment with fatty acids on permeation across rat skin. Skin Pharmacol Physiol. 2004;17:289-97.
11. Rastogi SK, Singh J. Effect of chemical penetration enhancer and iontophoresis on the in vitro percutaneous absorption enhancement of insulin through porcine epidermis. Pharm Dev Technol. 2005;10:97-104.

12. Wang Y, Fan Q, Song Y, Michniak B. Effects of fatty acids and iontophoresis on the delivery of midodrine hydrochloride and the structure of human skin. Pharm Res. 2003;20:1612-8.

13. Mina R, Melson P, Powell S, Rao M, Hinze C, Passo M et al. Effectiveness of dexamethasone iontophoresis for temporomandibular joint involvement in juvenile idiopathic arthritis. Arthritis Care Res (Hoboken). 2011;63:1511-6.

14. Garnock-Jones KP. Sumatriptan iontophoretic transdermal system: a review of its use in patients with acute migraine. Drugs. 2013;73:1483-90.

15. Power I. Fentanyl $\mathrm{HCl}$ iontophoretic transdermal system (ITS): clinical application of iontophoretic technology in the management of acute postoperative pain. Br J Anaesth. 2007;98:4-11.

16. Cheung E, Baerlocher MO, Asch M, Myers A. Venous access: a practical review for 2009. Can Fam Physician. 2009;55:494-6.

17. Priya B, Rashmi T, Bozena M. Transdermal Iontophoresis. Expert Opin Drug Deliv. 2006;3:127-38.

18. Prausnitz MR, Langer R. Transdermal drug delivery. Nat Biotechnol. 2008;26:1261-8.

19. Lino S, Muscarinic NY. M(2) acetylcholine receptor distribution in the guinea-pig gastrointestinal tract. Neuroscience. 2006;138:549-59. 